\title{
The Responses of Rat Trigeminal Ganglion Neurons to Capsaicin and Two Nonpungent Vanilloid Receptor Agonists, Olvanil and Glyceryl Nonamide
}

\author{
L. Liu, ${ }^{1}$ Y.-C. Lo, ${ }^{2}$ I.-J. Chen, ${ }^{1}$ and S. A. Simon ${ }^{1}$ \\ ${ }^{1}$ Departments of Neurobiology and Anesthesiology, Duke University Medical Center, Durham, North Carolina 27710 and \\ 2Department of Pharmacology, Kaohsiung, Taiwan, 80708 Republic of China
}

Capsaicin, the pungent ingredient in hot pepper, activates and subsequently desensitizes a subset of polymodal nociceptors. Because its initial application to skin produces pain, nonpungent analogs such as olvanil and glyceryl nonivamide (GLNVA) were synthesized to enhance its clinical use. To explore how these nonpungent analogs differ from capsaicin, whole-cell patch-clamp recordings were performed on cultured rat trigeminal ganglion neurons.

In neurons held at $-60 \mathrm{mV}$, capsaicin, olvanil, and GLNVA were found to activate one or two kinetically distinct inward currents. Two inward currents were also activated when extracellular $\mathrm{Ca}^{2+}$ was replaced with $\mathrm{Ba}^{2+}$ and also when intracellular chloride was replaced by aspartate. The reversal potentials of the rapidly and slowly activating currents were $15.3 \pm 6$ and $-4.0 \pm 2.5 \mathrm{mV}$, respectively. These data provide strong evidence for subtypes of vanilloid receptors. One difference among these agonists is that, on average, the activation kinetics of the currents evoked by $1 \mu \mathrm{m}$ olvanil and $30 \mu \mathrm{M}$ GLNVA are considerably slower than those evoked by $1 \mu \mathrm{M}$ capsaicin. Measurements of the peak current, Ip, versus agonist concentration were fit to the Hill equation to yield values of the half maximal concentrations $\left(K_{1 / 2}\right)$, and the Hill coefficients $(n)$. For capsaicin, olvanil, and GLNVA, $K_{1 / 2}=0.68,0.59$, and $27.0 \mu \mathrm{M}$; and $n=1.38$, 1.32, and 1.24, respectively.

We propose that olvanil and GLNVA are nonpungent because they activate different subtypes of receptors and/or because of their activation kinetics (compared with capsaicin) are, on average, slower than the rate they inhibit action potentials from polymodal nociceptors.

Key words: pain; taste; vanilloid receptors; pungent; olvanil; capsaicin
Capsaicin, the pungent ingredient in chili pepper, produces pain and inflammation when placed on skin or mucus membranes (Holzer, 1991; Szolcanyi et al., 1991). These effects result from the activation of cation-selective channels in polymodal nociceptors causing peptides and transmitters to be released from their peripheral and central terminals. The selectivity of capsaicin for polymodal nociceptors, coupled with the fact that on repeated applications it produces a long-lasting desensitization of these neurons, has made it useful clinically as an anti-nociceptive and -inflammatory compound. ${ }^{a}$

One disadvantage of using capsaicin clinically is that its initial contact with skin produces marked pain and inflammation that sometimes prevents continuance. To reduce these initial responses, protocols were developed in which the capsaicin concentration, the interstimulus interval, and the delivery vehicle were varied systematically (Craft and Porreca, 1992). Another approach was to synthesize analogs of capsaicin with properties that will not cause marked pain on the initial application. This latter approach has followed the pioneering structure activity work of

\footnotetext{
Received Dec. 20, 1996; revised March 4, 1997; accepted March 24, 1997.

This work was supported by National Institutes of Health Grant DC 01065 and by the Philip Morris Corporation.

Correspondence should be addressed to Dr. Sidney A. Simon, Department of Neurobiology, Duke University Medical Center, Durham, NC 27710.

Copyright (C) 1997 Society for Neuroscience $0270-6474 / 97 / 174101-11 \$ 05.00 / 0$
}

${ }^{a}$ In the literature describing the effects of capsaicin desensitization has meant the diminution of a response to capsaicin after the initial application. In this paper we will refer to this process as tachyphylaxis or rundown. Desensitization will refer to the diminution of the magnitude of the evoked current in the presence of an agonist.
Szolcsanyi and Jansco-Gabor (1975a,b), who found that analogs with longer acyl chains or with altered phenolic hydroxy groups were less pungent than capsaicin. Subsequently, numerous capsaicin analogs were synthesized with the goal of finding a compound that does not produce pain on its initial application, but that still retains its desensitizing characteristics.

The best characterized of the long-acyl chain, less-pungent capsaicin analogs is olvanil (Fig. 1). Olvanil, like capsaicin, has antinociceptive and -inflammatory properties (Brand et al., 1987), and is equipotent with capsaicin in its ability to increase $\mathrm{Ca}^{2+}$ influx in cultured rat DRGs (Walpole and Wrigglesworth, 1993), and in behavioral measurements of pain (Campbell et al., 1993). Moreover, whole-nerve recordings from rat spinal cord found that olvanil and capsaicin cross-desensitize, suggesting that they activate the same receptor (Dray et al., 1990). In this regard, preliminary patch-clamp data on DRG neurons that showed that the inward currents activated by $0.5 \mu \mathrm{M}$ olvanil or $0.5 \mu \mathrm{M}$ capsaicin had approximately the same magnitude and activation kinetics (Bevan and Docherty, 1993).

Not all responses evoked by olvanil and capsaicin, however, were found to be identical. For example, in whole-nerve recordings from rat spinal cord the response evoked by $2 \mu \mathrm{M}$ capsaicin was mimicked by $500 \mu \mathrm{M}$ olvanil (Dickenson et al., 1990; Dray et al., 1990). That is, olvanil was less potent than capsaicin in its ability to activate nociceptors. Also $2-10 \mu \mathrm{M}$ capsaicin, but not 2-10 $\mu \mathrm{M}$ olvanil, evoked the release of peptides from rat spinal cord (Dickenson et al., 1990). In contrast, olvanil was found to be 10 times more potent than capsaicin in increasing blood flow in rabbit skin (Hughes et al., 1992). Finally, olvanil and capsaicin 

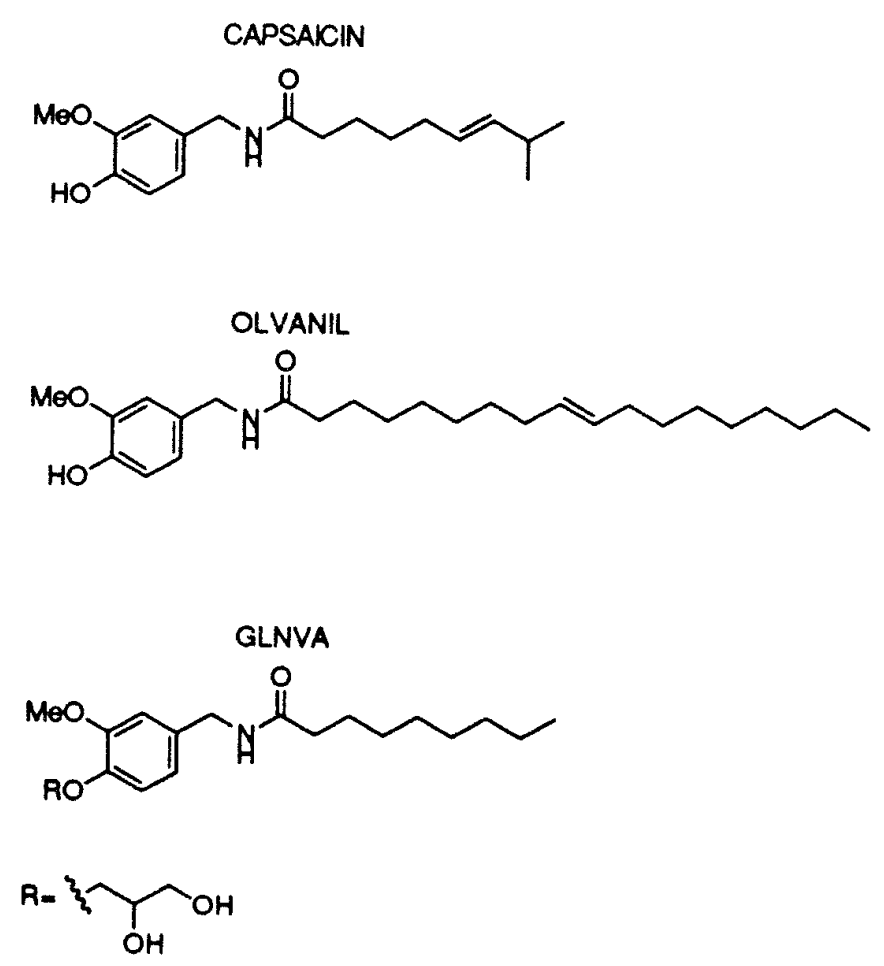

Figure 1. Chemical structures of capsaicin, olvanil, and GLNVA. For GLNVA, the phenolic hydroxy group of the synthetic capsaicin analog nonanoyl-vanillamide is replaced by a glycerol moiety.

differ in their ability to produce thermoregulatory desensitization (Brand et al., 1987). One rationalization of these data is that there are subtypes of capsaicin (vanilloid) receptors that respond differently to the same agonists (Szallasi, 1994; Szallasi et al., 1997; Appendino and Szallasi, 1997; Cruz et al., 1996).

Recently, glyceryl nonivamide (GLNVA, Fig. 1) another nonpungent, anti-nociceptive capsaicin analog was synthesized (Chen et al., 1992). Although GLNVA shares many similarities with capsaicin, there are significant differences in some of the physiological responses evoked by these compounds. For example, capsaicin elicits a triphasic blood pressure response, bradycardia, and apnea, whereas GLNVA elicited a monophasic reduction in blood pressure with little effect on heart rate and respiration (Yeh et al., 1993). The origins of these differences were not well understood.

To better understand how nonpungent capsaicin analogs differ from capsaicin, we measured the currents evoked by these three compounds from cultured rat trigeminal neurons.

\section{MATERIALS AND METHODS}

Materials. Salts were reagent grade. Unless otherwise stated, all other drugs and enzymes were purchased from Sigma (St. Louis, MO). Olvanil [N-(3-methyoxy-4-hydroxybenzyl) oleamide] was the generous gift of Dr. L. Brand of Proctor and Gamble (Cincinnati, OH). GLNVA was synthesized as described previously (Yeh et al., 1993).

Cell culture. Rat trigeminal cells were cultured as described previously (Liu and Simon, 1996a,c). Trigeminal ganglion cells were obtained from Sprague Dawley rats (150-250 gm) that were anesthetized with sodium pentobarbital $(50 \mathrm{mg} / \mathrm{kg})$. The cells were washed several times in a cold $\left(4^{\circ} \mathrm{C}\right)$ modified HBSS containing (in $\mathrm{mM}$ ): $\mathrm{NaCl} 130, \mathrm{KCl} 5, \mathrm{KH}_{2} \mathrm{PO}_{4} 0.3$, $\mathrm{NaHCO}_{3} 4.0, \mathrm{Na}_{2} \mathrm{HPO}_{4}$ 0.3, D-glucose 5.6, and HEPES 10. They were then incubated for $40 \mathrm{~min}$ at $37^{\circ} \mathrm{C}$ in HBSS containing $1 \mathrm{mg} / \mathrm{ml}$ collagenase (Type XI-S), triturated with a flamed Pasteur pipette to separate cells and remove processes, and finally incubated at $37^{\circ} \mathrm{C}$ for $8 \mathrm{~min}$ with $0.1-1.0 \mathrm{mg} / \mathrm{ml}$ DNase I (Type IV). Subsequently, they were retriturated and washed/centrifuged three times in F-14 medium (Life Technologies,
Gaithersburg, MD). They were then resuspended and placed in a Petri dish with F-14 containing 10\% fetal calf serum. The cultures were maintained in an incubator at $37^{\circ} \mathrm{C}$ equilibrated with $5 \% \mathrm{CO}_{2}$. Patchclamp experiments were performed on cells cultured for 12-24 hr. At the beginning of each experiment, the neurons were placed in a chamber containing Krebs-Henseleit (KH) buffer on an inverted microscope where the projected soma diameters were measured using a calibrated eyepiece. The composition (in $\mathrm{mm}$ ) of $\mathrm{KH}$ was: $\mathrm{NaCl} \mathrm{145,} \mathrm{KCl} 5, \mathrm{CaCl}_{2}$ $2.0, \mathrm{MgCl}_{2} 1.0$, HEPES 10 , and D-glucose $10, \mathrm{pH} 7.4$. Rat trigeminal ganglion neurons had a mean membrane potential of $-52 \mathrm{mV}$ (Liu et al., 1993). Experiments were performed at room temperature.

Patch clamp. The chamber containing the neurons had a volume of 500 $\mu \mathrm{l}$ and was perfused continuously by $\mathrm{KH}$ flowing into the chamber at a rate of $6 \mathrm{ml} / \mathrm{min}$ (Liu and Simon, 1996c). In addition, capsaicin, olvanil, GLNVA, and capsazepine (Tocris Cookson, St. Louis, MO.) were dissolved in $\mathrm{KH}$ and, if necessary, a small quantity of dimethylsulfoxide. These solutions were delivered to the cell using a multibarreled electrode (Adams and List Associated, Westbury, NY) placed $\sim 20-50 \mu \mathrm{m}$ from the cell by opening a valve. The solution in each barrel flowed at a rate of 6 $\mu \mathrm{l} / \mathrm{sec}$. The onset and removal times of the stimuli were obtained by event markers associated with the opening or closing of the valves. In experiments in which extracellular $\mathrm{BaCl}_{2}$ replaced $\mathrm{CaCl}_{2}$ (see below), the $\mathrm{KH}-\mathrm{Ca}$ buffer was replaced with a continuously perfusing $\mathrm{KH}-\mathrm{Ba}$ buffer to ensure that no calcium remained bathing the cell before the cell was reexposed to capsaicin in KH-Ba.

To test whether $10 \mu \mathrm{m}$ capsazepine would inhibit the currents activated by olvanil or GLNVA, as it does those of capsaicin (Liu and Simon, 1996c), these two agonists (in KH buffer) were initially applied to the neurons for $32 \mathrm{sec}$. After washing for $3 \mathrm{~min}, 10 \mu \mathrm{M}$ capsazepine was applied to the cell for $3 \mathrm{~min}$, and then a solution of capsazepine and olvanil or capsazepine and GLNVA was applied for $30 \mathrm{sec}$. To test for reversibility the cells were subsequently washed for 6 min with $\mathrm{KH}$ buffer, whereupon olvanil or GLNVA was reapplied. The percentage of blockage $(B)$, by capsazepine was calculated using $B=100 \%-\left(I \mathrm{p}_{\mathrm{cpz}} /\left(I \mathrm{p}_{1}+\right.\right.$ $\left.I \mathrm{p}_{2}\right) / 2$ ), where $I \mathrm{p}_{\mathrm{cpz}}$ is the peak current in the presence of capsazepine, and $I \mathrm{p}_{1}$ and $I \mathrm{p}_{2}$ are the peak currents of the first and second applications of the two agonists.

Patch-clamp recordings in the whole-cell configuration were performed with an Axoclamp 1D patch-clamp amplifier (Axon Instruments, Foster City, CA). The output was digitized with a Digidata 1200 A/D converter (Axon Instruments) and was sampled every $20 \mathrm{msec}$ unless stated otherwise. Series resistance was compensated at least $80 \%$, but leak currents were not. Electrode resistances were $\sim 2-5 \mathrm{M} \Omega$. In control experiments the microelectrode contained (in $\mathrm{mM}$ ): $\mathrm{KCl} 140, \mathrm{CaCl}_{2} 1.0, \mathrm{MgCl}_{2} 2.0$, BAPTA [1,2-bis(2-aminophenoyx)ethane- $N^{\prime}, N^{\prime}, N^{\prime}, N^{\prime}$-tetracetic acid] 10, HEPES 10, and $\mathrm{K}_{2}$-ATP 5 adjusted to $\mathrm{pH}$ 7.3. This solution was called SIS. To eliminate $\mathrm{Cl}^{-}$currents, in most experiments the internal solution contained (in mM): $\mathrm{K}$ aspartate $140, \mathrm{KCl} 1, \mathrm{CaSO}_{4} 0.2 \mathrm{H}_{2} 01, \mathrm{MgSO}_{4} 2$, BAPTA 10, HEPES 10 , and $\mathrm{K}_{2}$-ATP 5 adjusted to $\mathrm{pH}$ 7.3. This solution was called Kaspartate. In other experiments the $140 \mathrm{~mm} \mathrm{KCl}$ was replaced by $70 \mathrm{~mm} \mathrm{CsCl}$ and $70 \mathrm{~mm} \mathrm{CsF}$. This solution was called Cs-SIS. Finally, to guard against the possibility of capsaicin activating secondary $\mathrm{Ca}^{2+}$-dependent currents, in some experiments the extracellular solution contained $\mathrm{KH}$ with $2 \mathrm{mM} \mathrm{BaCl} 2$ replacing $2 \mathrm{mM} \mathrm{CaCl}_{2}$. This solution was called KH-Ba.

Current-voltage response. P-clamp software (Axon Instruments) was used to obtain all the current-voltage $(I-V)$ curves. In these experiments the extracellular solution contained KH plus $0.3 \mathrm{~mm}$ TTX. The intracellular solution contained SIS. The cells were held at either -70 or -80 $\mathrm{mV}$, and the current was ramped periodically to 70 or $80 \mathrm{mV}$ in times ranging from 0.2 to $2 \mathrm{sec}$. In most experiments the voltage cycle was 0.2 sec. The current was sampled every $0.375 \mathrm{msec}$. The ramp was applied before, during, and after agonist application. Because the amplifier sometimes saturated when the potential reached $80 \mathrm{mV}$, we present $I-V$ curves for the range $\pm 60 \mathrm{mV}$.

Tachyphylaxis experiments. Unless stated otherwise, after the application of capsaicin, olvanil, or GLNVA, the cell was washed for 6 min with $\mathrm{KH}$ buffer before reapplication of the agonists.

\section{RESULTS}

\section{Currents activated by capsaicin, olvanil, and GLNVA}

We have shown previously that in rat trigeminal ganglion neurons held at $-60 \mathrm{mV}$ and bathed in $\mathrm{KH} / \mathrm{SIS}$ buffers, $1 \mu \mathrm{M}$ capsaicin activated inward currents in $\sim 60 \%$ of the neurons (Liu and 


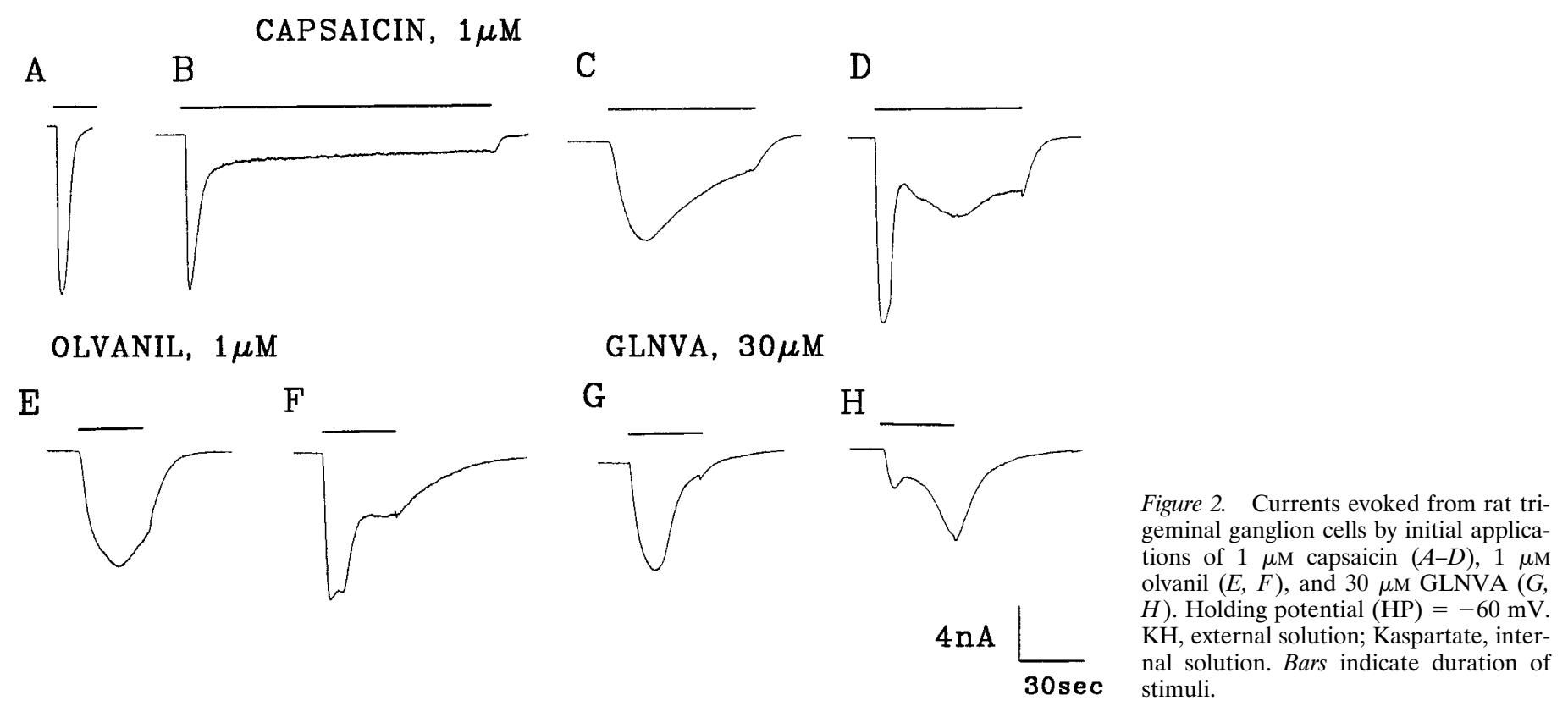

Simon, 1996c). Moreover, we found that capsaicin can activate one (Fig. $2 A-C$ ) or more kinetically distinct inward currents (Fig. $2 D$, Liu et al. 1996d). These inward currents can vary widely in their activation kinetics as characterized by the time to peak $(\tau p)$. Although most had peak times between 1 and $9 \sec (4.2 \pm 3.1$; mean \pm SD) (Liu and Simon, 1996c) others had peak times of $\sim 40 \mathrm{sec}(41.4 \pm 16.4 \mathrm{sec}$; mean \pm SD) (Liu and Simon, 1996b) (Fig. 2D). These currents also varied in their desensitization kinetics (compare Fig. $2 A-C$ ) and the extent to which they desensitized, which ranged from $100 \%$ (Fig. $2 A$ ) to not desensitizing (Liu and Simon, 1996c). Another common feature of the capsaicin-activated currents is that after the cell is washed, the current increases transiently before returning to baseline (Fig. $2 B-D)$. This transient increase is ascribed to a transition from a desensitized channel state to an open state before reaching a closed state (Liu and Simon, 1996c).

Under the same conditions, midrange concentrations (see below) of olvanil $(1 \mu \mathrm{M})$ and GLNVA $(30 \mu \mathrm{M})$ also activate inward currents. Approximately $64 \%$ of the cells were activated by $1 \mu \mathrm{M}$ olvanil (25/39) or $30 \mu \mathrm{M}$ GLNVA (26/41). Like capsaicin, olvanil may activate currents with one (Figs. $2 E, 4 A$ ) or two peaks (Figs. $2 F, 6 A, 9)$. The primary difference between capsaicin and olvanil is that the time to peak of the olvanil-induced currents was on average markedly longer, being $\tau p=25.2 \pm 8 \mathrm{sec}(n=25)$. Specifically, when $1 \mu \mathrm{M}$ olvanil was initially applied to a neuron, rapidly activating currents, such as seen in Figure $2 F$, were observed in only 3 of 25 cells. For these cells the mean $\tau p$ was $4.3 \mathrm{sec}$. The olvanil-induced currents desensitize and slowly return to baseline after wash (Fig. $2 E$ ). After washing, transient inward currents were observed frequently (Fig. $4 A$ ). Of the 25 cells that were activated by $1 \mu \mathrm{M}$ olvanil, none exhibited rapidly activating and completely desensitizing currents such as those shown in Figure $2 A$ for capsaicin.

The currents activated by $30 \mu \mathrm{M}$ GLNVA were similar to those induced by $1 \mu \mathrm{M}$ olvanil, in the sense that most $(24 / 26)$ of the GLNVA-induced currents activated slowly $(\tau p=26.6 \pm 11.4 \mathrm{sec}$; $n=26$ ). One example is shown in Figure $2 G$. In the remaining two neurons, the GLNVA-induced currents exhibited multiple peaks (Figs. $2 H, 8$ ). In these neurons the more rapidly activating current had a mean time to peak of $3.7 \mathrm{sec}$. Rapidly desensitizing currents, such as those seen in Figure $2 A, B$ for capsaicin, were never observed with $30 \mu \mathrm{M}$ GLNVA. In summary, at midrange concentrations, the currents activated by olvanil and GLNVA, on average, activate more slowly than those evoked by capsaicin.

When two inward currents are activated by a single agonist it is important to determine whether the more slowly activating current arises as a consequence of the activation of the more rapidly activating current. One possibility is that at $-60 \mathrm{mV}$ the secondary inward current could be a $\mathrm{Ca}^{2+}$-activated $\mathrm{Cl}^{-}$current. We have eliminated this possibility by first showing that two inward currents can also be activated when the intracellular solution contains K aspartate (Fig. 2) (Liu and Simon, 1996c). We now show that two inward currents can also be activated when $\mathrm{Ca}^{2+}$ in the $\mathrm{KH}$ buffer is replaced by $\mathrm{Ba}^{2+}(\mathrm{KH}-\mathrm{Ba})$. Figure 3 shows the results of an experiment in which $1 \mu \mathrm{M}$ capsaicin activated two distinct inward currents. After wash, reapplication of capsaicin (in $\mathrm{KH}-\mathrm{Ba}$ ) also evoked two inward currents, although the magnitude of these currents was smaller by $\sim 85 \%$. The partial reversibility of the currents eliminates the possibility that the inhibition was not entirely the result of "rundown" or tachyphylaxis (Figs. 9, 10). We found that replacement of $2 \mathrm{mM} \mathrm{Ca}^{2+}$ by $2 \mathrm{mM} \mathrm{Ba}^{2+}$ inhibited the rapid and slowly capsaicin-activated currents by $68 \pm 31 \%$ and $62 \pm 20 \%(n=8)$, respectively. Because $\mathrm{Ba}^{2+}$ cannot activate most $\mathrm{Ca}^{2+}$ - activated currents (Hille, 1993), these data suggest that the slowly activating current is not a $\mathrm{Ca}^{2+}$-activated current. In a subsequent publication we will show that $\mathrm{Ba}^{2+}$ blocks capsaicin-activated currents (Liu and Simon, unpublished observations).

\section{Capsazepine inhibits olvanil- and GLNVA-induced currents}

Capsazepine has been shown to be a specific and competitive inhibitor of capsaicin-induced responses (Bevan et al., 1992). To determine whether olvanil and GLNVA activate the same class of receptors as capsaicin we investigated whether the currents activated by these two agonists can also be inhibited by capsazepine. We considered initially the currents activated by olvanil. Figure $4 A$ shows a cell in which $10 \mu \mathrm{M}$ capsazepine partially inhibited the 
Figure 3. Barium reduces capsaicinactivated currents. Left trace, Capsaicin dissolved in $\mathrm{KH}$ buffer evoked two inward currents. After wash, $\mathrm{a} \mathrm{Ca}^{2+}$-free, but $\mathrm{Ba}^{2+}$-containing $\mathrm{KH}$ buffer $(\mathrm{KH}-$ $\mathrm{Ba}$ ) was exchanged for the $\mathrm{KH}$ buffer to rid the chamber of calcium. After a 3 min incubation period, capsaicin in $\mathrm{KH}-\mathrm{Ba}$ was reapplied (middle trace). After wash in $\mathrm{KH}$, reapplication of capsaicin in KH (right trace) partially reversed the inhibition by $\mathrm{Ba}^{2+}$. SIS, internal solution. $\mathrm{HP}=-60 \mathrm{mV}$. Bars indicate duration of stimuli.
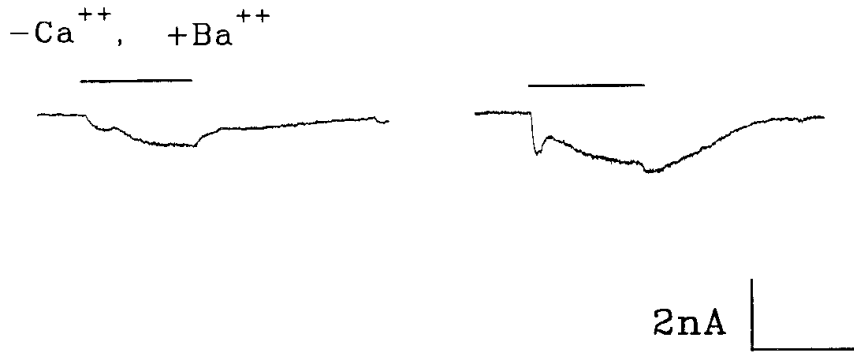

$60 \mathrm{sec}$ olvanil-induced current by $88 \%$. After wash, the magnitude of current increased after reapplication of olvanil, showing that the current was indeed inhibited by capsazepine. On average the olvanil-induced current was reduced $91.4 \pm 9.2 \%(n=6)$ by capsazepine. Complete reversibility of the olvanil-induced currents was never attained, most likely because of the relatively rapid olvanil-induced tachyphylaxis (Figs. 9, 10). The partial inhibition can be taken as evidence of multiple subtypes of vanilloid receptors.

The GLNVA-induced currents were reversibly inhibited by capsazepine (Fig. $4 B$ ). On average, $10 \mu \mathrm{M}$ capsazepine inhibited $94.5 \pm 7.5 \%(n=6)$ of the currents evoked by $30 \mu \mathrm{M}$ GLNVA.

\section{$\boldsymbol{I} \boldsymbol{V}$ relationships}

Capsaicin

$I-V$ relationships for capsaicin were obtained previously by applying it to cells at several different holding potentials. Between applications the cell was washed for 6-8 min. Using this protocol, $I-V$ plots were obtained that were slightly rectifying and that had reversal potentials of $\sim 4 \mathrm{mV}$ (Liu and Simon, 1996b,c). These small reversal potentials represent the slowly activated currents and indicate the presence of a nonselective cation channel (Oh et al., 1996). Although numerical values of the reversal potential of the rapidly activating current were not reported, it was nevertheless noted that it was more positive than $4 \mathrm{mV}$ (Liu and Simon, 1996c). The "repeated application" method has the advantage of knowing that the currents activated were those actually activated by capsaicin, but it has the disadvantage of not knowing how the magnitude and kinetics of the currents changed because of rundown (tachyphylaxis). Using the voltage-ramp method (see Materials and Methods) the full $I-V$ curve as well as the voltagedependent kinetics can be obtained with a single application. Consequently, problems associated with rundown or tachyphylaxis are avoided.

To obtain $I-V$ relationships and kinetics of the responses to capsaicin, voltage ramps were generated before, during, and after application of $1 \mu \mathrm{M}$ capsaicin. The currents activated "before" (even in the presence of $0.3 \mu \mathrm{M}$ TTX, $50 \mu \mathrm{M}$ verapamil, and intracellular $\mathrm{Cs}^{+}$) were frequently voltage-dependent (Fig. 5A). These voltage-dependent currents, however, were prominent only during the first 50 (or so) cycles whereupon they diminish after repeated cycling to an extent that the $I-V$ curve becomes smaller and approximately linear near the 100th cycle. At this juncture the background (BKG) $I-V$ curve was defined. The two $I-V$ plots labeled $a$ and $b$ in Figure $5 A$ were obtained at different times after
Figure 4. Capsazepine inhibits olvanil and GLNVA-induced currents. $A$, Olvanil $(1 \mu \mathrm{M})$ activates an inward current. After a 3 min wash with $\mathrm{KH}$, the cell was incubated with $10 \mu \mathrm{M}$ capsazepine in $\mathrm{KH}$ for $3 \mathrm{~min}$ before the application of $10 \mu \mathrm{M}$ capsazepine plus $1 \mu \mathrm{M}$ olvanil (middle trace). After a 6 min wash, olvanil was reapplied. $B$, Same as above except for $30 \mu \mathrm{M}$ GLNVA. HP $=-60 \mathrm{~m}$. Bars indicate duration of stimuli.
A OLVANIL, $1 \mu \mathrm{M}$

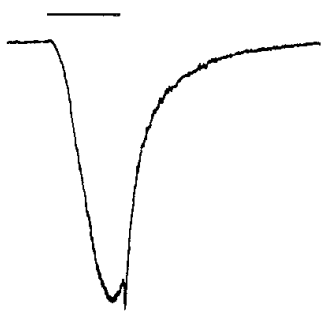

B GLNVA, $30 \mu \mathrm{M}$

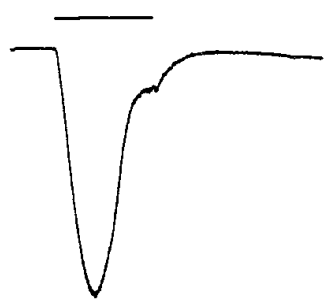

+CAPSAZEPINE, $10 \mu \mathrm{M}$
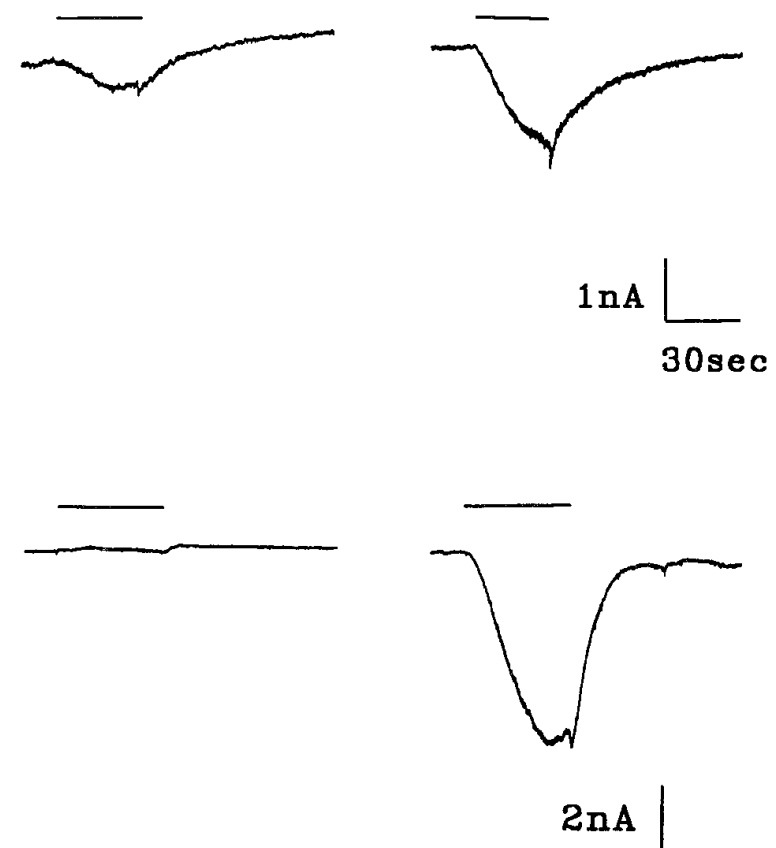
A
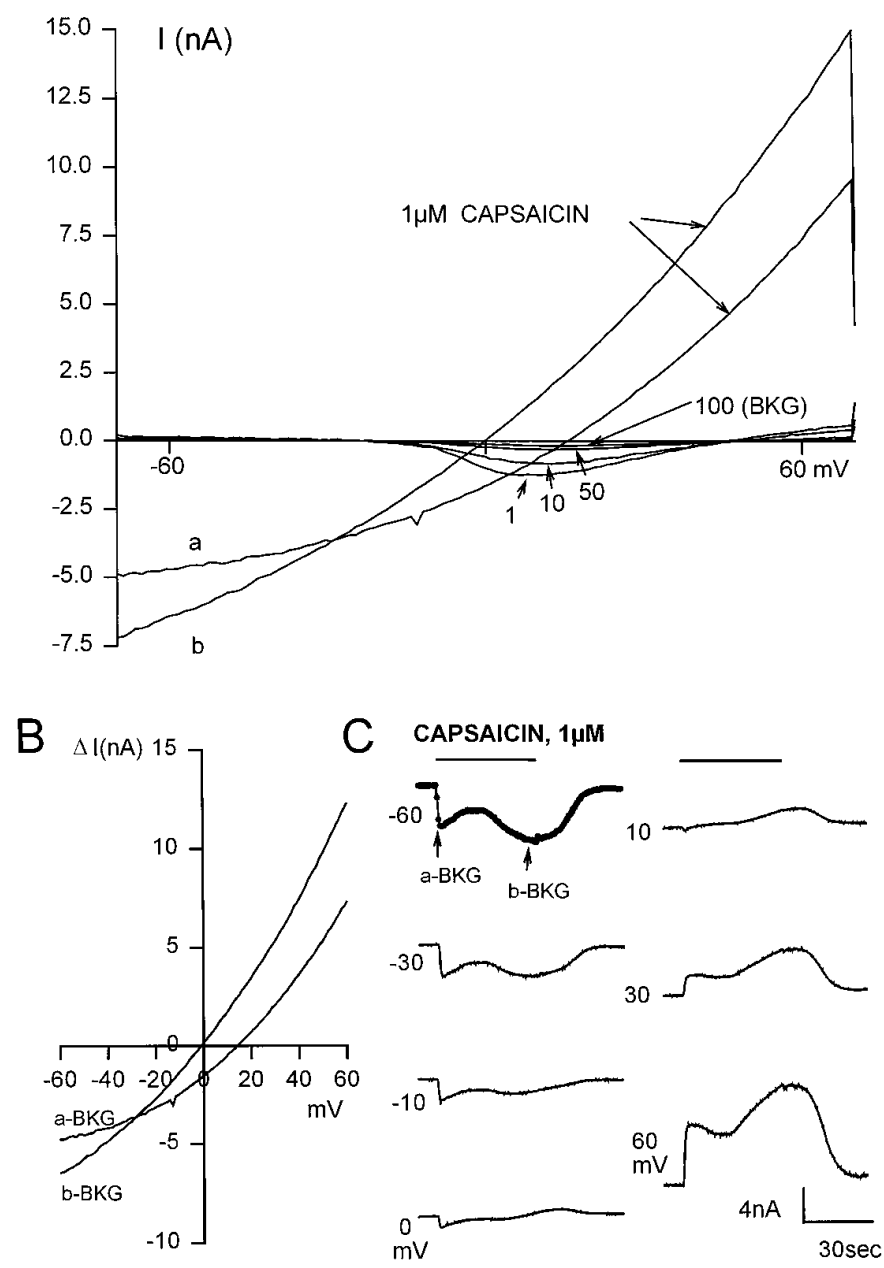

Figure 5. $I-V$ relationship using the voltage-ramp method. $A$, A voltage ramp from -70 to $70 \mathrm{mV}$ was applied every $0.2 \mathrm{sec}$ to a neuron before, during, and after application of $1 \mu \mathrm{M}$ capsaicin. Several of the 100 "before" traces were labeled. Note that the amplitude of the voltagedependent currents decreases with repeated cycling. Trace 100 was taken as the background current $(B K G)$. The curves labeled $a$ and $b$ were taken $\sim 4$ and $\sim 30 \mathrm{sec}$ after application of $1 \mu \mathrm{M}$ capsaicin. $B$, Plots of two of the current-voltage curves. $\Delta I$ is the measured current minus the BKG. The $a-B K G$ and $b-B K G$ labels refer to the two $I-V$ curves in $5 A$. $C, \Delta I$ versus time plots at seven different potentials that range from -60 to $+60 \mathrm{mV}$. The $-60 \mathrm{mV}$ trace was constructed from the currents at $-60 \mathrm{mV}$ from the family of $\Delta I-V$ curves shown in $5 B$. Each circle represents the current at $-60 \mathrm{mV}$ for one of the family of $\Delta I-V$ curves. In this figure every fourth data point is shown. For clarity, the six other $\Delta I$-time relationships are shown as continuous at $-60 \mathrm{mV}$. External solution, $\mathrm{KH}+0.3 \mu \mathrm{M}$ TTX. Internal solution, Cs-SIS. Bar indicates duration of stimulus.

the application of capsaicin, with $a$ being obtained at the earlier time. Figure $5 B$ shows a plot of $\Delta I$ versus $V$ where $\Delta I=I_{\text {cap }}-$ $I_{\mathrm{BKG}}$, where $I_{\mathrm{BKG}}$ is the background current obtained just before the application of capsaicin, and where $I_{\text {cap }}$ is the current obtained two times ( $a$ and $b$ ) after its application. It is important to note that the reversal potential for these two $\Delta I-V$ curves $a$ and $b$ are 14.5 and $0 \mathrm{mV}$, respectively. Note also that in both plots the outward currents are larger than the inward currents. The $I-V$ curve representing the slower activating current is the one commonly reported, because it has a reversal potential near $0 \mathrm{mV}$. For six cells in which two currents were activated by capsaicin, the reversal potentials obtained at the peaks of the rapidly and slowly activating currents were $15.3 \pm 6.0$ and $-4 \pm 2.5 \mathrm{mV}$, respectively. These values are significantly different $(p<0.05)$ using the paired $t$ test. Because the reversal potential of the rapidly activating current is greater than that of the slowly activating current, it follows that it is more cation-selective.

Using the data obtained from the entire family of $\Delta I-V$ curves, plots of $\Delta I$ versus time at different potentials could be generated. Figure $5 C$ shows such plots at seven different potentials (from -60 to $+60 \mathrm{mV}$ ) for $1 \mu \mathrm{M}$ capsaicin. The trace labeled $-60 \mathrm{mV}$ was constructed from the currents at $-60 \mathrm{mV}$ from the family of $\Delta I-V$ curves shown in Figure $5 B$. Each circle represents the current at $-60 \mathrm{mV}$ for one of the $\Delta I-V$ curves. The peak times for the fast and slow currents were 2.8 and $30 \mathrm{sec}$, respectively. These values are consistent with those obtained using the voltage-clamp method (Fig. 2). For clarity, the six other $\Delta I$-time relationships were shown as continuous. In summary, using the voltage-ramp method we found that capsaicin can activate two currents with kinetics and reversal potentials that are distinctly different, that the $I-V$ curves are nonlinear, and that the voltage-ramp method is a valid method to obtain the kinetic responses to capsaicin at different voltages.

\section{I-V plots for capsaicin, olvanil, and GLNVA}

Using the protocol described above we now present the results of an experiment (Fig. 6) in which $1 \mu \mathrm{M}$ olvanil, $1 \mu \mathrm{M}$ capsaicin, and $30 \mu \mathrm{M}$ GLNVA were applied consecutively to a neuron. Between applications the cell was washed for $6 \mathrm{~min}$. The currents generated by these three agonists (at $-60 \mathrm{mV}$ ) are shown in Figure $6 A$. The $\Delta I-V$ relationships taken at two "widely separated" times (Fig. $6 A$, arrows) are presented in Figure $6 B$. The envelope of the olvanil-induced currents, seen at $-60 \mathrm{mV}$, indicates the presence of two inward currents with peak times of $\sim 4$ and $\sim 18 \mathrm{sec}$, respectively. The $\Delta I-V$ curves corresponding to the peak of the rapidly activating current ( $a$ in Fig. $6 B$ ) and the recovery phase of the slower current ( $b$ in Fig. $6 B$ ) demonstrate clearly the existence of two distinct inward currents, because they have reversal potentials of 27 and $-5 \mathrm{mV}$ for the more rapidly and slowly activating currents, respectively. The application of capsaicin (middle trace) also evoked two currents with peak times of 4 and $\sim 13 \mathrm{sec}$. That two currents are present in this trace is confirmed by the $\Delta I-V$ curves where the reversal potentials of the more rapidly $a$ - and slowly $b$-activating currents were 25 and $-7 \mathrm{mV}$, respectively. This result is important because it shows that both compounds can activate the same currents. The application of $30 \mu \mathrm{M}$ GLNVA, evoked only a slowly activating and desensitizing current $(\tau p=18$ sec) that transiently increased after wash. The two $\Delta I-V$ curves obtained during the activation and desensitization phases had similar reversal potentials (approximately $-5 \mathrm{mV}$ ), indicating that GLNVA activated only a single current, the slowly activating current. Thus, it follows that either the rapidly activating current was desensitized by capsaicin, or that in this neuron the rapidly activating current was not activated by GLNVA. Whatever the case, it is clear from this experiment that the two currents are separable.

For cells in which these agonists evoke multiple currents, the $I-V$ curves and the reversal potentials are clearly comprised of two distinct contributions. Consequently, the numerical values obtained for the $I-V$ curves and the reversal potentials are not truly reflective of a single transport pathway. The reversal potentials for the rapidly activating response were $18 \mathrm{mV}$ (range, 4-35 mV), and those for the slowly activating induced were $\sim 5 \mathrm{mV}$ (range, 
A

A

OLVANIL, $1 \mu \mathrm{M}$

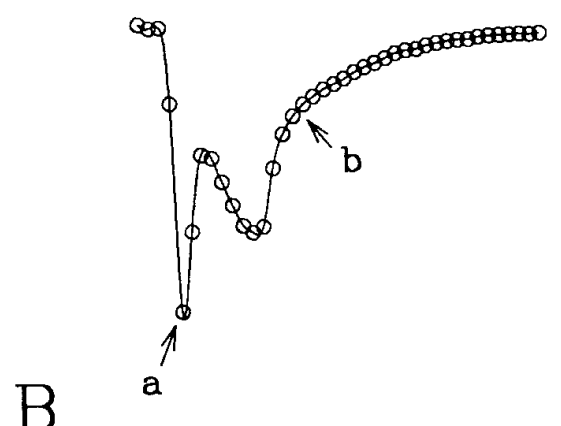

CAPSAICIN, $1 \mu \mathrm{M}$

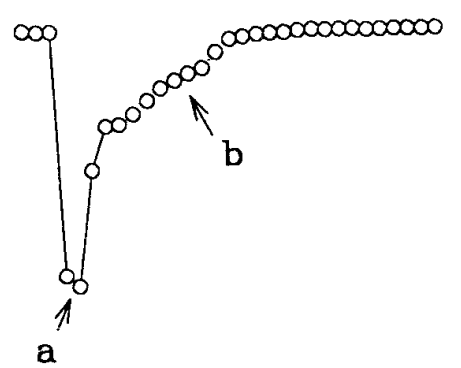

GLNVA, $30 \mu \mathrm{M}$

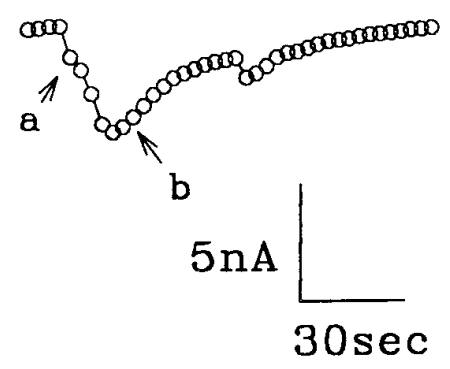

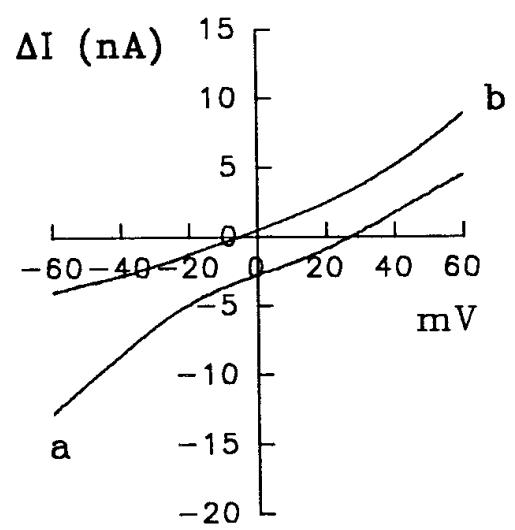
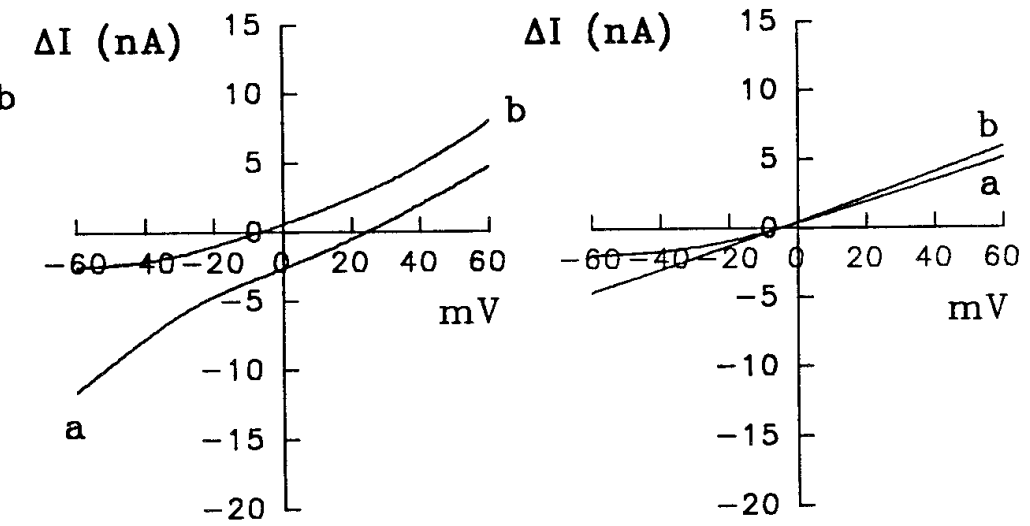

Figure 6. Current versus time responses at $-60 \mathrm{mV}$ for $1 \mu \mathrm{M}$ olvanil, $1 \mu \mathrm{M}$ capsaicin, and $30 \mu \mathrm{M}$ GLNVA obtained in the same neuron using the methods described in Figure 5. Between applications the cell was washed for $6 \mathrm{~min}$. $A$, The top traces show the currents obtained from the family of $\Delta I-V$ curves at $-60 \mathrm{mV}$. It is evident that olvanil and capsaicin evoke two inward currents, whereas GLNVA evokes a single slowly activating current. The labels $a$ and $b$ refer to the times the $\Delta I-V$ plots in $6 B$ were obtained. $B$. Two $\Delta I-V$ plots corresponding to the times shown in the top traces. Note that the reversal potential for GLNVA at the two different times is approximately the same. External solution, $\mathrm{KH}+0.3 \mu \mathrm{M}$ TTX; internal solution, SIS. Bar indicates duration of stimuli.

-5 to $11.5 \mathrm{mV})$. We take this heterogeneity in reversal potential to reflect different subtypes of vanilloid receptor channels.

\section{Dose-response for olvanil and GLNVA}

Figure 7 shows the responses of two rat trigeminal ganglion neurons held at $-60 \mathrm{mV}$ to increasing concentrations of olvanil and GLNVA. In these experiments olvanil and GLNVA was applied for 10 and $30 \mathrm{sec}$, respectively. Olvanil was applied for shorter times to reduce tachyphylaxis. Between applications the cells were washed for 6 min.

Olvanil did not evoke a response at $0.03 \mu \mathrm{M}$, but evoked a small inward current at $0.1 \mu \mathrm{M}$ (threshold). Increasing the olvanil concentration decreased the time to peak and increased the magnitude of the current until it remained unchanged with increasing concentration. The largest change in the magnitude of the currents occurred between 0.3 and $1 \mu \mathrm{M}$ olvanil.

A dose-response series for GLNVA is also shown in Figure 7. In this neuron, $0.3 \mu \mathrm{M}$ GLNVA did not evoke a current, $1 \mu \mathrm{M}$ GLNVA evoked a small inward current (threshold), and increasing the GLNVA concentration decreased the time to peak and increased the magnitude of the current until saturation. Note that the activation kinetics were slow even at $100 \mu \mathrm{M}$ GLNVA.

In constructing dose-response curves, the peak currents, Ip, are usually plotted against the agonist concentration. In constructing plots of Ip versus concentration for olvanil, however, we were aware that $10 \mathrm{sec}$ may not have been sufficiently long for the current to reach its absolute maximum (see trace for 0.3 $\mu \mathrm{M}$ olvanil). Nevertheless, to obtain some facsimile of the "true" dose-response plot, we had to choose conditions in which tachyphylaxis (Figs. 9, 10) is kept reasonably small and yet apply the agonist sufficiently long so that the currents increase with concentration. By averaging data from experiments such as those shown in Figure 7, we constructed doseresponse curves for capsaicin (Liu and Simon, 1996c), olvanil $(n=7)$, and GLNVA $(n=6)$. (For the seven olvanil experiments, four of them had data points for all six concentrations and three had data points for five concentrations.) These data, shown in Figure 8 , were then fit to the Hill equation, $I \mathrm{p} / I \mathrm{p}_{\max }$ $=1 /\left(1+\left(K_{1 / 2} / \mathrm{C}\right)^{\mathrm{n}}\right)$, where $K_{1 / 2}$ is the half maximal concentration, $n$ is the Hill coefficient, and $I \mathrm{p}_{\max }$ is the maximum current. For capsaicin, olvanil, and GLNVA, it was found that $I \mathrm{p}_{\max }=$ $7.10,7.19$, and $5.86 \mathrm{nA}$, respectively; $K_{1 / 2}=0.68,0.59$, and 27.0 $\mu \mathrm{M}$, respectively; and $n=1.38,1.32$, and 1.24 , respectively. The Ip-concentration plots for olvanil and capsaicin are indistinguishable. The parameters used to fit to the GLNVA data should be considered tentative, because the response profile did not, on average, reach saturation even at $100 \mu \mathrm{M}$.

\section{Tachyphylaxis}

Tachyphylaxis is defined as the diminution of a response to a drug on repeated applications. Because of the pioneering work of Jancso et al. (1967) many in vivo and in vitro studies have shown 
OLVANIL

$0.03 \mu \mathrm{M}-$

0.1

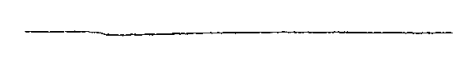

0.3

1

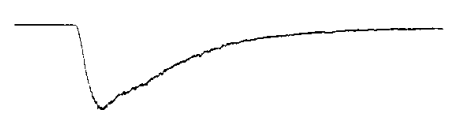

10

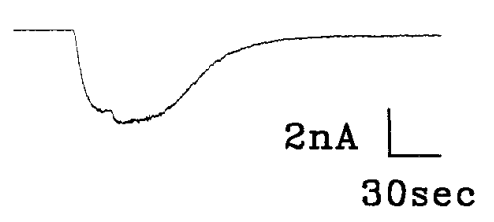

\section{GLNVA}

\section{$0.3 \mu \mathrm{M}$}

1

3

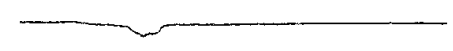

10

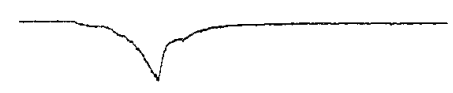

30

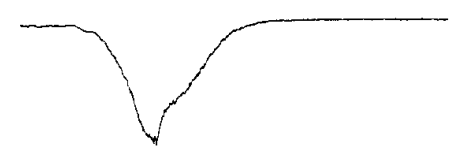

100

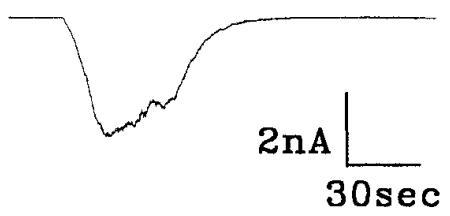

Figure 7. Dose-response for olvanil and GLNVA. Bar indicates duration of stimuli. $\mathrm{HP}=-60 \mathrm{mV}$. that capsaicin induces tachyphylaxis (Holzer, 1991; Liu and Simon, 1996b,c). In line with the rest of this paper, here we compare the tachyphylaxis produced by capsaicin (Liu and Simon, 1996b), olvanil, and GLNVA. In neurons held at $-60 \mathrm{mV}$, we found that $1 \mu \mathrm{M}$ olvanil (Fig. 9A) and $30 \mu \mathrm{M}$ GLNVA (Fig. 9B) produce tachyphylaxis. In these experiments, either $1 \mu \mathrm{M}$ olvanil or $30 \mu \mathrm{M}$ GLNVA were applied for $30 \mathrm{sec}$ up to seven times with each application separated by a $2 \mathrm{~min}, 30 \mathrm{sec}$ wash. The first olvanil application produced a response with a peak at $27.6 \mathrm{sec}$. The second application greatly reduced $I p$ by $81 \%$. Subsequent applications slightly reduced the current and by the seventh application the current was essentially eliminated. The decrease in current on repeated applications cannot be attributable entirely to "rundown" because much smaller decreases are seen with $1 \mu \mathrm{M}$ cap- saicin (Liu and Simon, 1996b) (Fig. 10), $100 \mu \mathrm{M}$ piperine (Liu and Simon, 1996b), and $30 \mu \mathrm{M}$ GLNVA (Figs. 9B, 10).

Figure $9 B$ shows the currents evoked by repeated applications of $30 \mu \mathrm{M}$ GLNVA. The initial GLNVA application induced a large inward current. After wash, the second application produced a current having a smaller magnitude and with slower activation kinetics. The third GLNVA application produced a small increase in the current, and the fourth to seventh applications produced approximately the same magnitude of currents.

The results of six individual tachyphylaxis experiments with 1 $\mu \mathrm{M}$ olvanil (neurons labeled O1-O6) and five with $30 \mu \mathrm{M}$ GLNVA (neurons labeled G1-G5) are shown in Figure 10 $A$. For example, the olvanil trace in Figure $9 A$ corresponds to olvanil neuron O3', and the GLNVA trace in Figure $9 B$ corresponds to GLNVA

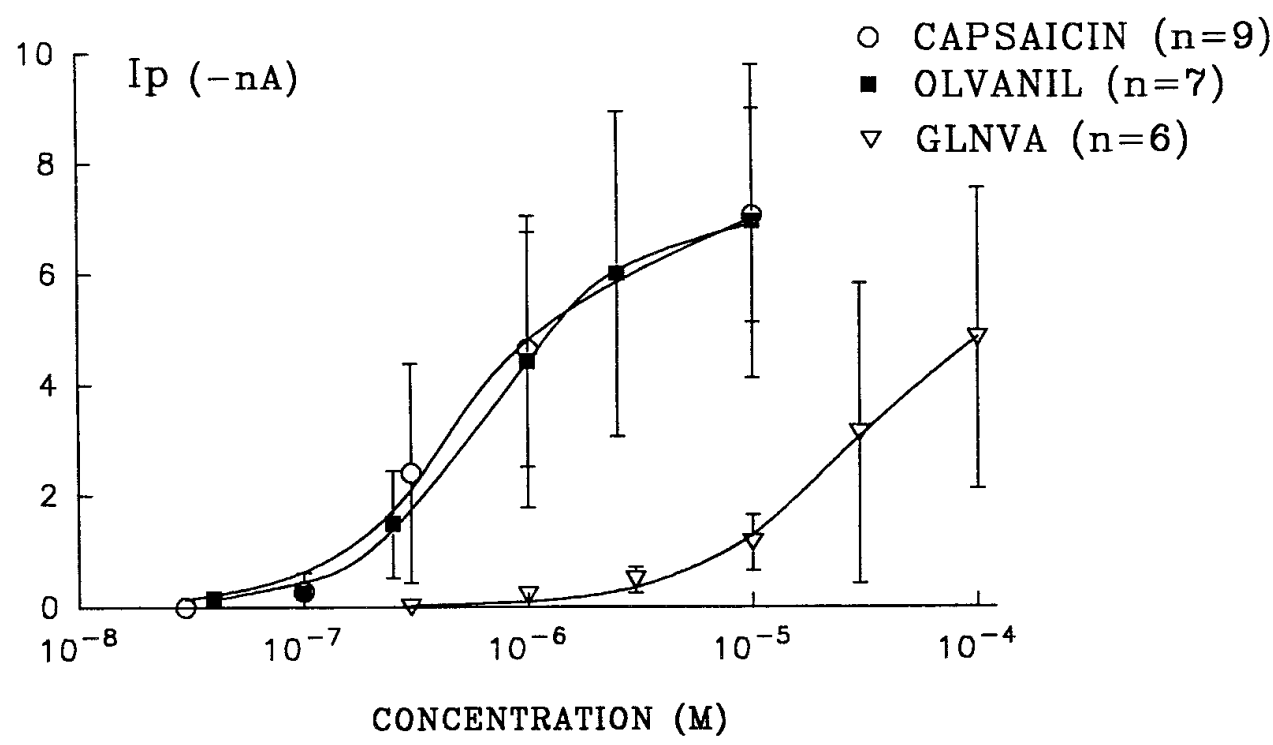

Figure 8. Dose-response for capsaicin, olvanil, and GLNVA obtained at a holding potential of $-60 \mathrm{mV}$. The data represent the mean $\pm \mathrm{SD}$ for capsaicin $(n=9)$, olvanil $(n=7)$, and GLNVA $(n=6)$. The solid lines were fit to the Hill equation (see text). $\mathrm{HP}=-60 \mathrm{mV}$. The capsaicin data were from Liu and Simon (1996c). 


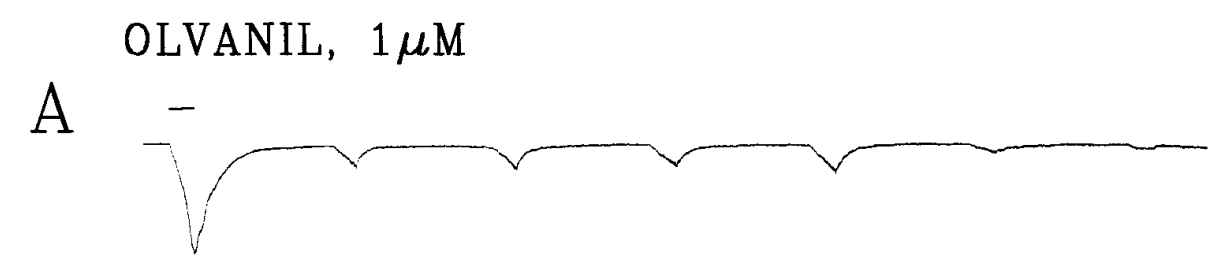

Figure 9. Tachyphylaxis of olvanil and GLNVA. Currents evoked by seven repeated $30 \mathrm{sec}$ applications of $1 \mu \mathrm{M}$ olvanil $(A)$ and $30 \mu \mathrm{M}$ GLNVA $(B)$ in $\mathrm{KH}$ buffer. Between applications the cells were washed $2 \mathrm{~min} 30 \mathrm{sec}$ with $\mathrm{KH}$ buffer. HP $=-60 \mathrm{mV}$. Bar indicates

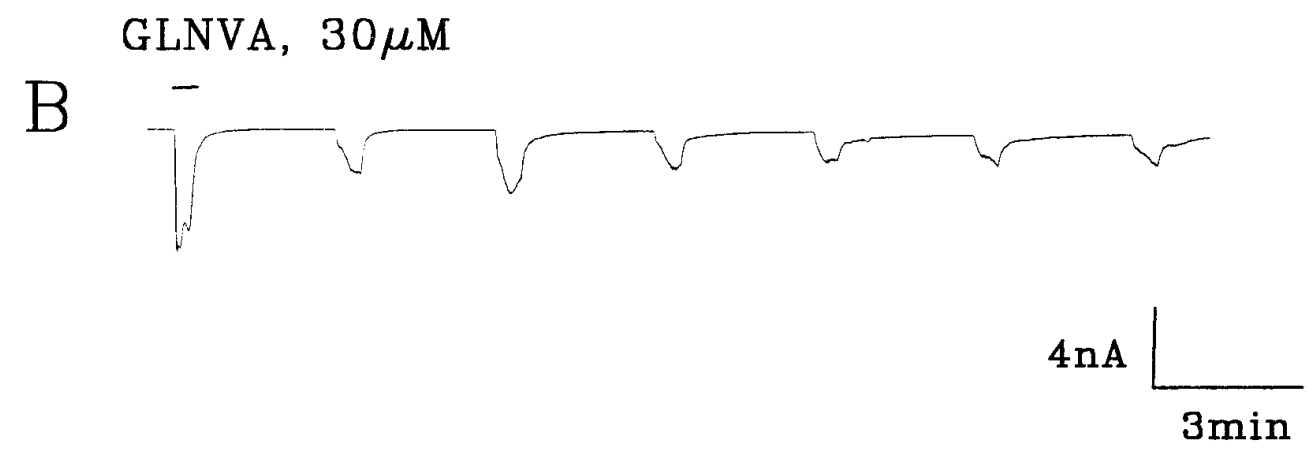
duration of stimuli.
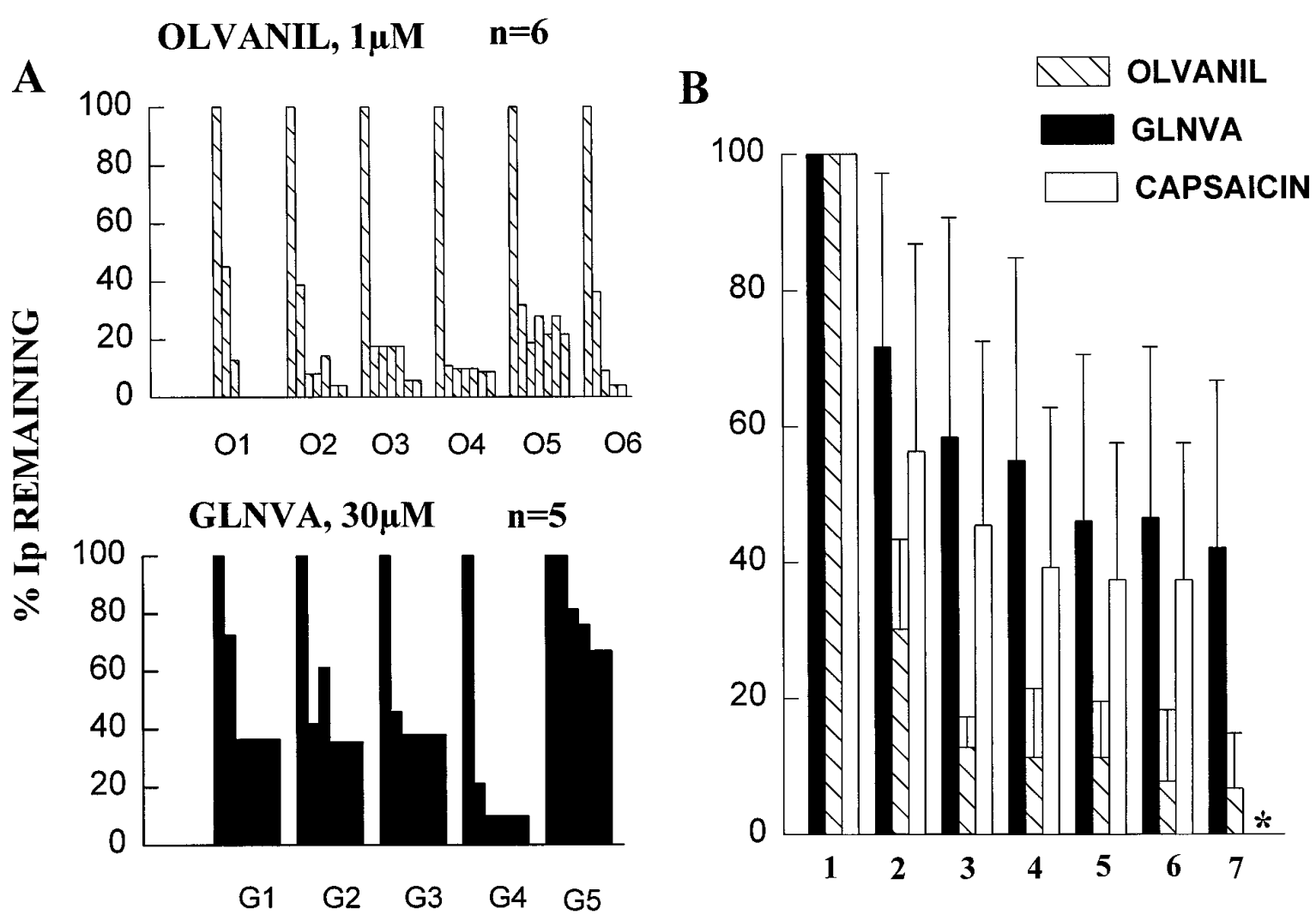

Figure 10. Results of tachyphylaxis experiments. The results of six olvanil (O1-O6) and five GLNVA (G1-G5) experiments are presented to illustrate the heterogeneity of the responses. $A$, Plots of the \%Ip remaining with respect to the initial application (=100\%). For example, for olvanil neuron "O1" the current disappeared completely after the third application. B, The mean $\pm \mathrm{SD}$ of the data presented in Figure $10 A$. The asterisk for the seventh application of GLNVA data are presented because in two of the five experiments the cell died. Capsaicin data are from Liu and Simon (1996b).

neuron $\mathrm{G}^{\prime}$. The data are presented in this manner to illustrate the heterogeneity of the tachyphylaxis responses. For example, olvanil neuron $\mathrm{O} 1$ exhibited complete tachyphylaxis after three applications, whereas for olvanil neuron O5, $20 \%$ of the initial current could be evoked on the seventh application. In contrast, for GLNVA the current was never completely inhibited after six applications. The means of these responses, together with those obtained previously for $1 \mu \mathrm{M}$ capsaicin (Liu and Simon, 1996c), are shown in Figure $10 B$. These data can be fit to the relation $\% I \mathrm{p}$ remaining $=A \exp (-\mathrm{n} \times 3 \min / \tau)+B$, where $A+B=100 \%$, $t$ is the time constant of the current that did not recover in a $2 \mathrm{~min}$, 30 sec wash, and $n=0,1,2$. The constant $B$, reflects the 
percentage of the current that can be recovered after washing for $2.5 \mathrm{~min}$. Thus, for $1 \mu \mathrm{M}$ olvanil the data fit the equation $\% \mathrm{Ip}$ remaining $=89 \% \exp (-n \times 3 \mathrm{~min} / 2.27 \mathrm{~min})+7.5 \% ;(r=0.997)$. For $30 \mu \mathrm{M}$ GLNVA the data fit $\%$ Ip remaining $=62.2 \%$ $\exp (-n \times 3 \mathrm{~min} / 2.85 \mathrm{~min})+37.3 \% ;(r=0.998)$, and for capsaicin we found previously $\%$ Ip remaining $=56.8 \% \exp (-n \times 3$ $\min / 4.83 \mathrm{~min})+42.6 \%$; $(r=0.994)$. In summary, for GLNVA and capsaicin the processes of rundown or tachyphylaxis are quite similar, whereas for olvanil tachyphylaxis is much more pronounced.

\section{DISCUSSION}

To understand the differences between capsaicin and two of its nonpungent analogs, olvanil and GLNVA, whole-cell patch-clamp studies were performed on rat trigeminal ganglion neurons. It was found that: (1) these compounds can activate two currents that can be distinguished by their reversal potentials and kinetics, (2) the major difference between them is that the currents activated by the nonpungent analogs have, on average, slower activation kinetics, and (3) the tachyphylaxis produced by olvanil is much greater than tachyphylaxis produced by either capsaicin or GLNVA.

\section{Olvanil and GLNVA belong to the family of compounds that activate vanilloid receptors}

Because olvanil and GLNVA are synthetic analogs of capsaicin they should mimic many of its physiological effects. Because these analogs were designed with the goal of being less or nonpungent, however, it should not be expected that they would mimic all the physiological responses of capsaicin with the exception of pungency, because the properties that go into making a compound less pungent may also alter other responses. The data obtained on cultured trigeminal ganglion neurons provide some reasons for the similar and different physiological responses evoked by these compounds. These three agonists are similar in that they activate inward currents in a subset of trigeminal ganglion neurons, are inhibited by capsazepine, can activate currents in the same cell, and can activate two distinct currents with similar reversal potentials in the same cell (Figs. 2-6). These data suggest that the similarity in their physiological responses comes from them activating some of the same subtypes of vanilloid receptors.

\section{Capsaicin activates distinct currents in trigeminal ganglion neurons}

There is good evidence for the existence of multiple subtypes of vanilloid receptors. These include the following: (1) binding studies of radiolabeled resiniferatoxin (RTX) that showed intraspecies heterogeneity and interspecies differences, (2) differences in the association constants and Hill coefficients in central and peripheral vanilloid receptors (Szallazi and Blumberg, 1993; Szallazi et al., 1993, 1994, 1995), and (3) vanilloid receptors that could be distinguished by their ability to induce vasoconstriction and/or be inhibited by capsazepine or ruthenium red (Colquhoun et al., 1995).

Our finding that the rapid and slowly activated currents are independent and have distinct reversal potentials as well as the existence of capsazepine-insensitive currents is good evidence for multiple subtypes of vanilloid receptors. These data also suggest that, like nicotinic acetylcholine and GABA receptors (McGehee and Role, 1995), different subtypes of vanilloid receptors may coexist in an individual neuron.

\section{Dose-response characteristics}

Olvanil was found to be equipotent to capsaicin in increasing $\mathrm{Ca}^{2+}$ influx in rat DRGs (Walpole and Wrigglesworth, 1993) and in behavioral measurements of pain (Campbell et al., 1993). That olvanil and capsaicin exhibit similar dose-response curves (Fig. 8) is consistent with these studies. Olvanil, however, was found to be 10 times more potent than capsaicin as a vasodilator (Hughes et al., 1992) and $\sim 250$ times less potent in evoking similar responses from rat spinal cord (Dray et al., 1990). These differences likely indicate the presence of subtypes of vanilloid receptors. GLNVA was shown to be $\sim 15$ times less potent than capsaicin in blood pressure measurements obtained from rats (Yeh et al., 1993). From the dose-response curves (Fig. 8) we found a 39-fold difference in the $K_{1 / 2}$ between capsaicin and GLNVA. Given the different nature of these measurements, we consider the agreement to be reasonable.

\section{Tachyphylaxis}

Although there have been many tachyphylaxis experiments performed that characterize the effects of capsaicin (Holzer, 1991), there is very little data regarding tachyphylaxis for olvanil and GLNVA. This study represents the first patch-clamp data describing tachyphylaxis (rundown) for these two nonpungent agonists. We found that the tachyphylaxis experiments could all fit to the equation $\%$ Ip remaining $=A \exp (-n \times 3 \mathrm{~min} / \tau)+B$, where $A+$ $B=100 \%, B$ is the percentage of the current that can be recovered after seven or more $2.5 \mathrm{~min}$ washes, and $A$ is the magnitude of the current that was not recovered. We have interpreted $A$ to represent the percentage of the channels that convert into a stable long-lived desensitized state with time constant, $\tau$, and $B$ the current that can be recovered after a $30 \mathrm{sec}$ application and a 2.5 min wash (Liu and Simon, 1996c). Whatever the processes underlying this behavior, the relative magnitudes of $A$ and $B$ for five capsazepine-inhibitable vanilloid receptor agonists (capsaicin, piperine, zingerone, olvanil, and GLNVA) can be compared. We found $B=42,7.5,37,60$, and $0 \%$ for $1 \mu \mathrm{M}$ capsaicin, $1 \mu \mathrm{M}$ olvanil, $30 \mu \mathrm{M}$ GLNVA, $100 \mu \mathrm{M}$ piperine, and $30 \mathrm{~mm}$ zingerone (Liu and Simon, 1996b,c), respectively. The $B$ values fall broadly into two groups: one containing olvanil and zingerone where little or none of the current recovered from a desensitized state, and the other containing capsaicin, GLNVA, and piperine, where approximately half the current recovered. Because capsaicin, piperine, and zingerone are pungent, whereas olvanil and GLNVA are nonpungent, it follows that the extent that a particular agonist induces tachyphylaxis (or recovers from desensitized states) is not correlated with its pungency.

\section{Speculations regarding the basis of pungency of capsaicin and its analogs}

Despite the fact that capsaicin, olvanil, and GLNVA activate the same classes of receptors they do not always produce the same physiological responses. As noted previously, one or both of these compounds may differ from capsaicin in potency, thermoregulatory effects, peptide release, blood pressure lowering, and pungency. Although there are several reasons why olvanil and GLNVA may give different physiological responses than capsaicin, such as the possibility that capsaicin acts peripherally whereas olvanil acts centrally (Dickenson et al., 1990), our data suggest that the differences between these two nonpungent analogs and capsaicin may be accounted for by either the presence and distribution of different receptor subtypes and/or by the slower rate of activation of the currents evoked by GLNVA and olvanil.

Pungency arises as a consequence of the activation of primary nociceptors that subsequently transmit information to the higher CNS centers. In the presence of depolarizing currents such as 
those produced by capsaicin, subsets of nociceptors evoke action potentials (Kenins, 1982) and thus transmit information to the CNS. If the subtypes of vanilloid receptors for nonpungent ana$\operatorname{logs}$ on the periphery are smaller affinities $\left(K_{1 / 2}\right)$ than those that are centrally located, then the probability of them being activated at the same concentration as capsaicin to produce nociception will be markedly reduced, and hence they will be less pungent.

Transmission of action potentials to the CNS (and hence pungency), however, could also be inhibited by blocking and/or inactivating voltage-dependent $\mathrm{Na}^{+}, \mathrm{K}^{+}$, and $\mathrm{Ca}^{2+}$ channels, because these channels are responsible for the generation, propagation, and transmission of action potentials. It is established that capsaicin blocks voltage-dependent $\mathrm{Na}^{+}, \mathrm{K}^{+}$, and $\mathrm{Ca}^{2+}$ channels (Marsh et al., 1987; Docherty et al., 1991; Kehl, 1994), which will prevent the generation of trains of action potentials. Despite these "local anesthetic" effects, capsaicin is pungent, meaning that action potentials are transmitted to the higher centers and that in primary nociceptors, depolarization precedes inhibition. To identify a compound that will not evoke action potentials from nociceptors, and still desensitize them, it would be advantageous to find a capsaicin analog that preferentially activates slowly activatable subtypes of vanilloid receptors while rapidly (relatively) inhibiting and/or inactivating voltage-dependent $\mathrm{Na}^{+}, \mathrm{K}^{+}$, and $\mathrm{Ca}^{2+}$ channels. [In this regard it is important to note that it may take several action potentials to get transmitter release to secondary neurons (Zucker and Haydon, 1988).] We propose that some of the differences between nonpungent and pungent compounds can be attributed to rates of activation of vanilloid receptors to the relative rates of blocking or inactivating of voltage-dependent $\mathrm{Na}^{+}, \mathrm{K}^{+}$, and $\mathrm{Ca}^{2+}$ channels.

We now focus on the interactions of olvanil with neurons because more is known about it than about the interactions of GLNVA. There is no question that olvanil inhibits $\mathrm{Na}^{+}$channels because it blocks evoked responses from $\mathrm{A} \beta$-fibers (Dickenson et al., 1990). The comparatively slower activation kinetics seen in most neurons with olvanil cannot be attributed to different $K_{1 / 2}$ values, because on average they were the same as the capsaicin values (Fig. 9). The generally slower activation kinetics of olvanil compared with capsaicin may rationalize why larger olvanil concentrations are required to evoke responses from rat spinal cords even though olvanil and capsaicin have similar antinociceptive potencies (Campbell et al., 1989; Dray et al., 1990). We suggest that until a sufficiently large olvanil concentration in spinal is cord used, such that the activation rates of olvanil become similar to those of capsaicin, its anesthetic effects will dominate, and under these conditions olvanil will be nonpungent.

Differences in the kinetics of various channel states is also seen by the greater tachyphylaxis produced by olvanil than by capsaicin (Fig. 10). This behavior likely reflects the longer time the channel spends in a desensitized state with olvanil. This explanation may also shed light on some properties of the ultrapotent agonist RTX that were previously unexplained. It was found that RTX is 20,000 times as potent as capsaicin in blocking neurogenic inflammation, but only 10 times as pungent, as determined in eye-wiping experiments (Szallasi and Blumberg, 1990). Because RTX, like olvanil, exhibits slow activation kinetics (Winter et al., 1990; Liu and Simon, 1996c) the relatively small pungency of RTX may be a consequence of its relatively rapid blocking or inactivating of voltagedependent channels and hence action potentials.

\section{REFERENCES}

Appendino G, Szallasi A (1997) Euphorbium: modern research on its active principle, resiniferatoxin, revives an ancient medicine. Life Sci, in press.

Bevan S, Hothi S, Hughes G, James IF, Rang HP, Shah K, Walpole CSJ, Yeats JC (1992) Capsazepine: a competitive antagonist of the sensory neurone excitant capsaicin. Br J Pharmacol 107:544-552.

Bevan S, Docherty RJ (1993) Cellular mechanisms of the action of capsaicin. In: Capsaicin in the study of pain (Wood J, ed), pp 27-44. London: Academic.

Brand L, Berman E, Schwen R, Loomans M, Janusz J, Bohne R, Maddin C, Gardner J, Lahann T, Farmer R, Jones L, Chiabrando C, Fanelli R (1987) NE-19550: a novel, orally active anti-inflammatory analgesic. Drugs Expl Clin Res X111:259-265.

Campbell E, Dray A, Perkins MN (1989) Comparison of capsaicin and olvanil as antinociceptive agents in vivo and in vitro. $\mathrm{Br} \mathrm{J}$ Pharmacol 98:907.

Campbell E, Bevan S, Dray A (1993) Clinical applications of capsaicin and its analogues. In: Capsaicin in the study of pain (Wood J, ed), pp 259-265. London: Academic.

Chen IJ, Yang JM, Yeh JL, Wu NB, Lo YC, Chen JS (1992) Hypotensive and antinociceptive effects of ether-linked and relatively nonpungent analogues of $N$-nonanoyl vanillyamide. Eur J Med Chem 27:187-192.

Colquhoun WQ, Eldershaw TPD, Bennett KL, Hall JH, Dora KA, Clark MG (1995) Functional and metabolic evidence for two different vanilloid (VN1 and VN2) receptors in rat perfused hindlimb. Life Sci 57:91-102.

Craft RM, Porreca F (1992) Treatment parameters of desensitization to capsaicin. Life Sci 51:1767-1775.

Cruz F, Avelino A, Coimbra A (1996) Desensitization follows excitation of bladder primary afferents by intravesical capsaicin, as shown by c-fos activation in rat spinal cord. Pain 64:553-557.

Dickenson A, Hughes C, Rueff A, Dray A (1990) A spinal mechanism of action is involved in the antinociception produced by the capsaicin analogue NE 19950 (olvanil). Pain 43:353-362.

Docherty RJ, Robertson B, Bevan S (1991) Capsaicin causes prolonged inhibition of voltage-activated calcium currents in adult dorsal root ganglion neurons in culture. Neuroscience 40:513-521.

Dray A, Bettany J, Reuff C, Walpole C, Wrigglesworth R (1990) NE19550 and NE-21610, antinociceptive capsaicin analogues: studies on nociceptive fibers of the neonatal rat tail in vitro. Eur $\mathbf{J}$ Pharmacol 181:289-293.

Hille B (1993) Ionic channels of excitable membranes. Sunderland, MA: Sinauer.

Holzer P (1991) Capsaicin: cellular targets, mechanisms of action, and selectivity for thin sensory neurons. Pharmacol Rev 43:143-201.

Hughes SR, Buckley TL, Brain SD (1992) Olvanil: more potent than capsaicin in stimulating the efferent function of sensory nerves. Eur J Pharmacol 219:481-484.

Jancso N, Jancso-Gabor A, Szolcsanyi J (1967) Direct evidence for neurogenic inflammation and its prevention by denervation and by pretreatment with capsaicin. Br J Chemother 31:138-151.

Kehl SJ (1994) Block of capsaicin of voltage-gated $\mathrm{K}^{+}$currents in melantrophs of rat pituitary. Br J Pharmacol 112:616-624.

Kenins P (1982) Responses of single nerve fibers to capsaicin applied to the skin. Neurosci Lett 29:83-88.

Liu L, Ma H, Pugh W, Simon SA (1993) Identification of acetylcholine receptors in adult rat trigeminal ganglion neurons. Brain Res 617:37-42.

Liu L, Simon SA (1996a) Capsaicin and nicotine both activate a subset of rat trigeminal ganglion neurons. Am J Physiol 270:C1807-C1814.

Liu L, Simon SA (1996b) Similarities and differences in the currents activated by capsaicin, piperine and zingerone in rat trigeminal ganglion cells. J Neurophysiol 76:1858-1869.

Liu L, Simon SA (1996c) Capsaicin-induced currents with distinct desensitization and $\mathrm{Ca}^{++}$dependence in rat trigeminal ganglion cells. J Neurophysiol 75:1503-1514.

Liu L, Wang Y, Simon SA (1996d) Capsaicin activated currents in rat dorsal root ganglion cells. Pain 64:191-195.

Marsh SJ, Stanefeld CE, Brown DA, Davey R, McCarthy D (1987) The mechanism and action of capsaicin on sensory C-type neurons and their axons in vitro. Neuroscience 23:275-289.

McGehee DS, Role LW (1995) Physiological diversity of nicotinic ace- 
tylcholine receptors expressed by vertebrate neurons. Annu Rev Physiol 57:521-546.

Oh U, Hwang SW, Kim D (1996) Capsaicin activates a nonselective cation channel in cultured neonatal dorsal root ganglion neurons. J Neurosci 16:1659-1667.

Szallasi A (1994) The vanilloid (capsaicin) receptor: receptor types and species differences. Gen Pharmacol 25:223-243.

Szallasi A, Nilsson S, Farkas-Szallasi T, Blumberg P, Hokfelt T, Lundberg JM (1995) Vanilloid (capsaicin) receptors in the rat: distribution in the brain, regional differences in the spinal cord, axonal transport to the periphery, and depletion by systemic vanilloid treatment. Brain Res 703:175-183.

Szallasi A, Blumberg PM (1990) Resiniferatoxin and its analogues provide novel insights into the pharmacology of the vanilloid (capsaicin) receptor. Life Sci 47:1399-1408.

Szallazi A, Lewin NA, Blumberg PM (1993) Vanilloid (capsaicin) receptor in the rat: positive cooperativity of resiniferatoxin binding and its modification by reduction and oxidation. J Pharmacol Exp Ther 256:678-683.

Szallazi A, Blumberg PM (1993) $\left[{ }^{3} \mathrm{H}\right]$ resiniferatoxin binding by the vanilloid receptor: species-related differences. Naunyn Schmiedebergs Arch Pharmacol 347:84-91.
Szolcanyi J, Bartho L, Petho G (1991) Capsaicin-sensitive bronchopulmonary receptors with dual sensory-efferent function: mode of action of capsaicin antagonists. Acta Physiol Hung 77:293-304.

Szolcanyi J, Jansco-Gabor A (1975a) Sensory effects of capsaicin congeners. II. Importance of chemical structure and pungency in desensitizing activity of capsaicin-type compounds. Arzneimittelforschung 25:33-37.

Szolcanyi J, Jansco-Gabor A (1975b) Sensory effects of capsaicin congeners. I. Relationship between chemical structure and pain producing potency of pungent agents. Arzneimittelforschung 25:1877-1881.

Walpole CSJ, Wrigglesworth R (1993) Structural requirements for capsaicin agonists and antagonists. In: Capsaicin in the study of pain (Wood J, ed), pp 63-81. London: Academic.

Winter J, Dray A, Wood JN, Yeats JC, Bevan S (1990) Cellular mechanism of action of resiniferatoxin: a potent sensory neuron excitotoxin. Brain Res 520:131-140.

Yeh J, Lo Y, Wang Y, Chen I (1993) Cardiovascular interactions of nonivamide, glycerol nonivamide, capsaicin analogues, substance $\mathrm{P}$ antagonist in rats. Brain Res Bull 30:641-648.

Zucker RS, Haydon PG (1988) Membrane potential has no direct role in evoking neurotransmitter release. Nature 335:360-362. 